
Brown's recovery was inspirational for researchers contemplating CCR5 as a target for genome editing. “There aren't many genes that I'm aware of where knocking them out doesn't do any harm, but instead has a therapeutic benefit," says Paula Cannon, a specialist in gene therapy and infectious disease at the University of Southern California in Los Angeles, who began her research of ZFNs as a tool for modifying CCR5 in 2007.

\section{INTO THE CLINIC}

The early success of SB-728 in replicating the CCR5 mutation, coupled with the story of the Berlin patient, as Brown became known, made researchers optimistic for clinical trials of the therapy. Between 2011 and 2013, researchers at the University of Pennsylvania, including immunotherapist Carl June and HIV specialist Pablo Tebas, used SB-728 to modify the genomes of helper $\mathrm{T}$ cells (the main target of HIV) obtained from 12 volunteers who were HIV positive. The researchers then cultivated the cells and transplanted them back into the donors. All the patients experienced a boost in their T-cell count, and each patient established a small, but stable subpopulation of immune cells with edited CCR5 genes. When treatment with antiretroviral drugs was interrupted to test whether the gene edits worked on their own, some patients saw transient reductions in their viral load (P. Tebas et al. N. Engl. J. Med. 370, 901-910; 2014). "The take-home for me was that the engineered cells got into patients and lasted longer than were expected," says Cannon, who was not directly involved in the study.

The next challenge was how to make this immune protection more potent and durable. One approach is to generate a larger population of ZFN-modified T cells. So, in a separate study, three patients were given a mild dose of chemotherapy to reduce their immunecell populations before transplantation. As an added boost to the therapy, in addition to editing helper T cells, the researchers also used SB-728 to modify killer T cells, which can also be destroyed by HIV infection.

"By creating a little more space and relying on the homeostatic factors that maintain T-cell levels, the cells have a better chance of survival and of giving rise to long-lasting cell populations," explains Riley. Two of the three patients experienced a profound drop in viral load, and they have not had to take antiretroviral treatment for more than a year.

How many cells must have their CCR5 genes edited to keep HIV at bay is not clear, however. About $5 \%$ of circulating T cells were successfully edited in the most recent trials, but Cannon points out that there are

also populations of T cells hiding in tissues, which makes the total pool a lot bigger than estimates from circulating cells would suggest. A fully modified T-cell population would be a tall order, but it may be possible to achieve protection even with a relatively small proportion of edited cells, according to HansPeter Kiem, a gene-therapy researcher at the Fred Hutchinson Cancer Research Center in Seattle, Washington. Kiem's group uses primate models to study the clinical potential of genome-edited immune cells. "If we only protect about $20 \%$ of the cells, we get a very robust boost in the immune response against HIV," says Kiem, referring to a 2013 study in which he tested the extent to which genetically modified stem cells protect pig-tailed macaques from simian HIV.

\section{LOOKING AHEAD}

Kiem thinks that the critical factor for building immunity against HIV is engraftment - the extent to which transplanted cells incorporate themselves into the tissues of the recipient's body. He and Cannon are separately exploring whether SB-728 might perform better if it is applied to haematopoietic stem cells - the common precursor of all of the various blood and immune cell sub-

"The talke-home for me was that the engineered cells got into patients and lasted longer than were expected." types - rather than to a few varieties of fully developed immune cells. "Then we can hit the T cells as well as monocytes, macrophages and other cell types that can be infected by, or serve as reservoirs for, HIV," explains Kiem.

However, stem cells are more difficult to cultivate and edit than T cells, and must be carefully maintained to ensure that they retain their developmental flexibility. Using stem cells also means more serious side effects for patients, who will have to undergo an aggressive course of chemotherapeutic 'conditioning' before treatment. "It kills some of the stem cells in the bone marrow to make room for the engineered cells - and it's not a trivial thing to undergo," cautions Cannon. This strategy is also slower to have an effect: it takes between six months and a year before the stem cells fully replenish the mature T-cell population. Cannon is involved with a newly launched clinical trial at the City of Hope Hospital in Duarte, California, which aims to explore how well these cells engraft into the bone marrow of 12 patients with HIV, and how many HIV-proof immune cells they each produce.

The therapeutic landscape for HIV has changed significantly in the ten years since Sangamo began pursuing this project. For a start, many patients can now keep their viral loads in check indefinitely by taking standard antiretrovirals. Nonetheless, a significant minority do not respond to these drugs.

Dale Ando, Sangamo's chief medical officer, is intrigued by the potential for a 'one-hit' treatment as opposed to having to take lifelong medication. "With antiretroviral therapy, there is a significant toll on the brain and heart, and increased risk of cancer, as well as chronic inflammation from long-term HIV infection," he says. By comparison, and leaving aside the effects of the associated chemotherapies, SB-728 has not been linked to any serious side effects. So far, all the data on SB-728 have assuaged the most immediate concerns about ZFNs - that off-target edits elsewhere in the genome may have damaging or carcinogenic consequences.

Perhaps more importantly, these HIV studies have helped to clear a regulatory path for future genome-editing therapeutic programmes. "We've had multiple discussions on T cells, stem cells and in vivo genome editing, so the US Food and Drug Administration (FDA) is quite comfortable," says Ando. As mainstream attention shifts to another genome-editing technology, CRISPR-Cas9, many believe that the FDA will find itself on familiar turf when drug applications that use the newer tool are filed.

From Cannon's perspective, much of the credit for this rapid progress belongs to the HIV patient community, whose political activism and hunger for a cure has helped to push genome editing into the clinic. "They've gotten us to this stage with this new therapy very quickly," she says, "and hopefully it will have benefits for all sorts of other diseases in the future."

Michael Eisenstein is a freelance writer based in Philadelphia, Pennsylvania. 